\title{
Synthesis and Studies on Non-Linear Optical Property of New Chalcone Based Polymers
}

\author{
T. S. PERUNDEVI ${ }^{1}$, D. REUBEN JONATHAN ${ }^{2}$ and S. KOTHAI ${ }^{* 1}$ \\ ${ }^{1}$ PG \& Research Department of Chemistry, Ethiraj College for Women, \\ Chennai-600 008, India \\ ${ }^{2}$ Department of Chemistry, Madras Christian College, Chennai-600 059, India \\ tsperundevi@yahoo.co.in
}

Received 8 December 2016 / Accepted 21 December 2016

\begin{abstract}
A new series of non linear optical (NLO) copolyesters have been synthesized by solution polycondensation method. The two diols namely 2,6 bis(4-methoxy-3-hydroxybenzylidene)-3methylcyclohexanone and 1-(3-hydroxyphenyl)-3-(4-methoxy-3-hydroxyphenyl)-prop-2-en-1-one, isopthaloyl chloride and succinyl, oxalyl and glutaryl chlorides were employed in the synthesis of the six copolyesters. Claisen-Schmidt condensation reaction was employed in synthesizing these two monomer diols. The formed polymeric materials were characterized by involving viscosity measurements, solubility study and FT-IR, ${ }^{1} \mathrm{H}$ NMR, ${ }^{13} \mathrm{C}$ NMR and UV-Visible spectroscopic techniques. Thermal transitions of these six copolyesters were recorded in an inert nitrogen atmosphere using Thermogravimetric Analysis. The second harmonic generation (SHG) efficiency of these copolyesters was measured by the standard powder technique using Nd-YAG laser and they were found to be NLO active.
\end{abstract}

Keywords: Chalcones, Copolyesters, Nonlinear optics, SHG efficiency

\section{Introduction}

Nonlinear optics has fascinated scientific community associated with information technology around the world due to its role in telecommunications, optical switching, sensor protections, and potential for optical information storage and coupling ${ }^{1}$. In order to design devices for such applications, it is mandatory to zero-in on either readily available materials or to go in for new materials that exhibit the desired nonlinear optical properties. Materials for nonlinear optics have been developed considerably in recent years, with organo-metallic complexes, composite glasses embedded with metal nanoparticles, novel semiconductors, and organic polymers ${ }^{2}$. There is a sincere demand to design and develop novel nonlinear materials with large molecular two photon absorption cross-sections to meet the present demand. However, such attempts toward understanding the NLO properties of organic materials are very limited ${ }^{3}$. A wide variety of materials have been investigated for nonlinear 
optics, among which organic materials are extremely attractive due to their prospective optical and electronic properties which can be certainly tailored by bringing about structural modification ${ }^{4}$.

Survey of literature indicates that organic materials with nonlinear optical properties will turn out to be key elements for future photonic technologies 5 . This may be due to superior and more rapid response of organic materials, their synthetic flexibility, a strong donor-acceptor intermolecular interaction and a delocalized $\pi$-electron system which facilitates nonlinear optical activity ${ }^{6,7}$. In addition to all this, the organic materials are also attractive due to their properties such as lower dielectric constant, better characteristics for industrial processing and flexibility in fitting the NLO effectiveness through the chemical modification of a given system ${ }^{8}$.

Chalcones have received substantial attention as materials for second-order nonlinear optical applications due to their ability to crystallize in non centrosymmetric structure. These emerged as promising candidates for nonlinear optics because of their noticeable nonlinearity and good optical power limiting properties ${ }^{9,10}$. Since the chalcone backbone is an asymmetric transmitter, it strongly increases the molecular nonlinearity for the electron donating and withdrawing group substitutions. Generally in charge transfer compounds, large nonlinear electronic polarization arises due to the large dipole moment change from ground state to an excited state by optical radiation ${ }^{11}$. The other advantage of chalcone molecules is that it offers greater flexibility in adopting suitable design strategies ${ }^{12,13}$ and thereby enhancing the nonlinear optical coefficients.

Chalcones and their derivatives are typically known as organic push-pull molecules consisting of polarizable bridge capped by electron donor and acceptor substituents. They also offer the required configuration meant for NLO activity with two planar benzene rings connected through a conjugated double bond ${ }^{14,15}$.

Poornesh et al., ${ }^{16}$ have studied nonlinear optical parameters of two bischalcone molecules in PMMA matrix using Z-scan technique. The measurement indicated that these bischalcones exhibit negative nonlinear refractive index. Hence, the bischalcone derivatives investigated by them can be a very hopeful class of materials for future photonic and optoelectronic applications.

To date, the molecular basis for the NLO properties is well established and the ease with which various polymers can be synthesized and chemically modified through rational design has led to the development of various systems to meet the optical characteristics required for fabrication of integrated optical devices.

\section{Experimental}

Isovanillin, 3-methylcyclohexanone and 3-hydroxyacetophenone were procured from Sigma-Aldrich and were utilized as such without any further purification. Sigma-Aldrich samples of isopthaloyl, succinyl, glutaryl and oxalyl chlorides were purchased and used for the copolymerization technique. SD-Fine AR sample of dimethylacetamide (DMAc) was used as such as a solvent for determining the inherent viscosity of the copolyester in solution. Spectral grade DMSO-d6 (Aldrich) was used having TMS as internal standard for recording NMR Spectra.

\section{Synthesis of chalcone diols}

The monomer diols namely 2,6-bis(4-methoxy-3-hydroxybenzylidene)-3-methylcyclohexanone, (IVCH) and 1-(3-hydroxyphenyl)-3-(4-methoxy-3-hydroxyphenyl)-prop-2-en-1-one (IVAP) were synthesized by the process reported by Chitra and coworkers ${ }^{17}$. 


\section{Synthesis of monomer diol IVCH}

Brown crystals of monomer diols were synthesized by the condensation of isovanillin and 3-methyl cyclohexanone in the mole ratio 2:1 as shown in the Scheme 1.<smiles>COc1ccc(C=O)cc1[OH+]</smiles><smiles>COc1ccc(/C=C2\C(=O)/C(=C/c3ccc(OC)c(O)c3)CCC2C)cc1O</smiles>

Scheme 1. Synthesis of monomer diol IVCH

Dry $\mathrm{HCl}$ gas was passed for about one hour through a well-cooled and stirred solution of 3-methyl cyclohexanone $(30 \mathrm{mmol})$ and isovanillin $(60 \mathrm{mmol})$ in $50 \mathrm{~mL}$ of dry methanol. Brown crystals of IVCH separated out. It was washed with double-distilled water and recrystallized from hot methanol. Melting point: $150{ }^{\circ} \mathrm{C}$ and $\%$ yield: $72 \%$. FT- IR (KBr) 3457 (b, O-H), 1650 (s, C=O) $\mathrm{cm}^{-1} ;{ }^{1} \mathrm{H}$ NMR (DMSO-d6): $\delta 9.1$ (s, 2H, -OH), $\delta 7.5-8.2$ (m, $12 \mathrm{H}$, aromatic), $\delta 6.7-6.9(\mathrm{dd}, 2 \mathrm{H},-\mathrm{CH}=\mathrm{CH}-)$.

\section{Preparation of monomer diol IVAP}

Yellow crystals of monomer diol were synthesized by the condensation of isovanillin and 3-hydroxyacetophenone in the mole ratio 1:1 as shown in the Scheme 2.<smiles>COc1ccc(C=O)cc1[OH+]</smiles><smiles>CC(=O)c1cccc(O)c1</smiles>

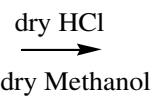

dry Methanol<smiles>COc1ccc(/C=C/C(=O)c2cccc(O)c2)cc1O</smiles>

Scheme 2. Synthesis of monomer diol IVAP

Dry $\mathrm{HCl}$ gas was passed for about 60 minutes through a well-cooled and stirred solution of 3-hydroxy acetophenone $(30 \mathrm{mmol})$ and isovanillin $(30 \mathrm{mmol})$ in $50 \mathrm{~mL}$ of dry methanol. Characteristic yellow coloured crystals of IVAP separated out. It was washed with doubledistilled water and recrystallized from hot methanol. Melting point: $168{ }^{\circ} \mathrm{C}$ and $\%$ yield: 87.6\%; FT-IR (KBr) 3457 (b, O-H), $1650(\mathrm{~s}, \mathrm{C}=\mathrm{O}) \mathrm{cm}^{-1} ;{ }^{1} \mathrm{H}$ NMR (DMSO-d6) $\delta 7.72\left(\mathrm{H}_{\beta}\right)$, $\delta 7.06\left(\mathrm{H}_{\alpha}\right), \delta$ 7.06-7.76 (Ar. H), $\delta 3.95\left(\mathrm{H}\right.$ of $\left.-\mathrm{OCH}_{3}\right), \delta 9.84(\mathrm{H}$ of $\mathrm{OH})$.

\section{Synthesis of copolyesters}

The procedure for the synthesis of typical copolyester is given here ${ }^{18}$. The monomer diol IVCH (1 mmol.) was dissolved in $10 \mathrm{~mL}$ of dry dimethyl formamide (DMF) taken in a $100 \mathrm{~mL}$ roundbottomed flask. After 5 minutes $1 \mathrm{~mL}$ of triethylamine was added and stirred well at room temperature for a time span of about 15 minutes in inert nitrogen atmosphere. Then succinyl chloride $(0.5 \mathrm{mmol}$.) and isopthaloyl chloride $(0.5 \mathrm{mmol})$ dissolved in $10 \mathrm{~mL}$ of DMF was added with constant stirring. Then the temperature was raised to $100{ }^{\circ} \mathrm{C}$ and maintained at this temperature with continuous stirring for a span of 3 hours. Finally when the reaction mixture was cooled to room temperature it was poured into $100 \mathrm{~mL}$ of methanol when the formed copolyester was precipitated. It was filtered, washed with methanol and dried in vacuum.

On similar lines the other five copolyesters were also synthesized. The diacid chlorides, diol-I and diol-II used together with the copolyester code of the six copolyesters formed are presented in Table 1. 
Table 1. List of monomers used, copolyester code of the six copolyesters, percentage of yield and inherent viscosities

\begin{tabular}{|c|c|c|c|c|}
\hline \multicolumn{2}{|c|}{$\begin{array}{l}\text { Common Diacid chloride : Isopthaloyl } \\
\text { chloride }\end{array}$} & \multirow[t]{2}{*}{ Copolyester } & \multirow[t]{2}{*}{ Yield } & \multirow[t]{2}{*}{ Viscosity } \\
\hline Diol & Diacid chloride II & & & \\
\hline IVCH & Succinyl chloride & PCSI & 72.65 & 1.04 \\
\hline IVCH & Glutaryl chloride & PCGI & 75.23 & 1.01 \\
\hline IVCH & Oxalyl chloide & PCOI & 78.58 & 1.00 \\
\hline IVAP & Succinyl chloride & PHSI & 86.21 & 1.06 \\
\hline IVAP & Glutaryl chloride & PHGI & 88.73 & 1.02 \\
\hline IVAP & Oxalyl chloide & PHOI & 92.84 & 1.00 \\
\hline
\end{tabular}

\section{Results and Discussion}

The six copolyesters synthesized by solution polycondensation method were characterized by involving analytical methods like, viscometry, solubility study, spectroscopy (FT-IR, ${ }^{1} \mathrm{H}$ NMR, ${ }^{13} \mathrm{C}$ NMR and UV-Visible) and TGA analysis.

\section{Solubility}

All the six copolyesters synthesized were tested qualitatively for their solubility in about nine common organic solvents and the results of their solubility are presented in Table 2.

Table 2. Solubility of copolyesters in common organic solvents

\begin{tabular}{cccccccccc}
\hline Copolyester & $\mathrm{C}_{6} \mathrm{H}_{12}$ & $\mathrm{C}_{6} \mathrm{H}_{6}$ & $\mathrm{CHCl}_{3}$ & $\mathrm{EtOAc}$ & $\left(\mathrm{CH}_{3}\right)_{2} \mathrm{CO}$ & $\mathrm{CH}_{3} \mathrm{OH}$ & DMAc & DMF & DMSO \\
\hline PCSI & -- & -- & +- & +- & +- & -- & ++ & ++ & ++ \\
PCGI & -- & -- & +- & +- & +- & -- & ++ & ++ & ++ \\
PCOI & -- & -- & +- & +- & +- & -- & ++ & ++ & ++ \\
PHSI & -- & -- & +- & +- & +- & -- & ++ & ++ & ++ \\
PHGI & -- & -- & +- & +- & +- & -- & ++ & ++ & ++ \\
PHOI & -- & -- & +- & +- & +- & -- & ++ & ++ & ++ \\
\hline
\end{tabular}

From the Table 2 it could be inferred that these copolyesters were thoroughly soluble in polar aprotic solvents such as dimethylsulphoxide, dimethylacetamide and dimethylformamide, partly soluble in moderately polar solvents like tetrahydrofuran and acetone but absolutely insoluble in least polar solvents like benzene and hexane. This might be attributed to the inter-molecular interactions of polar solvents with that of the ester linkage of present in the copolyester involved ${ }^{19}$.

\section{Viscosity}

The inherent viscosity of the resulting copolyesters was determined in dimethyl acetamide solution at $30{ }^{\circ} \mathrm{C}$ using Ubbelohde viscometer. In each case $25 \mathrm{mg}$ of pure dry copolyester sample was dissolved in $25 \mathrm{~mL}$ of DMAc, kept aside for some time with occasional shaking. The $\eta_{\text {inh }}$ was calculated from the flow time measurements. Usually an increase in the length of the spacer group increases the value of inherent viscosity which may be probably due to increase in the molecular weight of the copolyester ${ }^{20}$. Hence copolyesters with oxalyl group have low viscosity values.

\section{FT-IR Spectral study}

FT-IR spectrum of all the six copolyesters was recorded using Shimadzu FT-IR instrument. The FT-IR spectrum of the copolyester PHSI is given in Figure 1. 


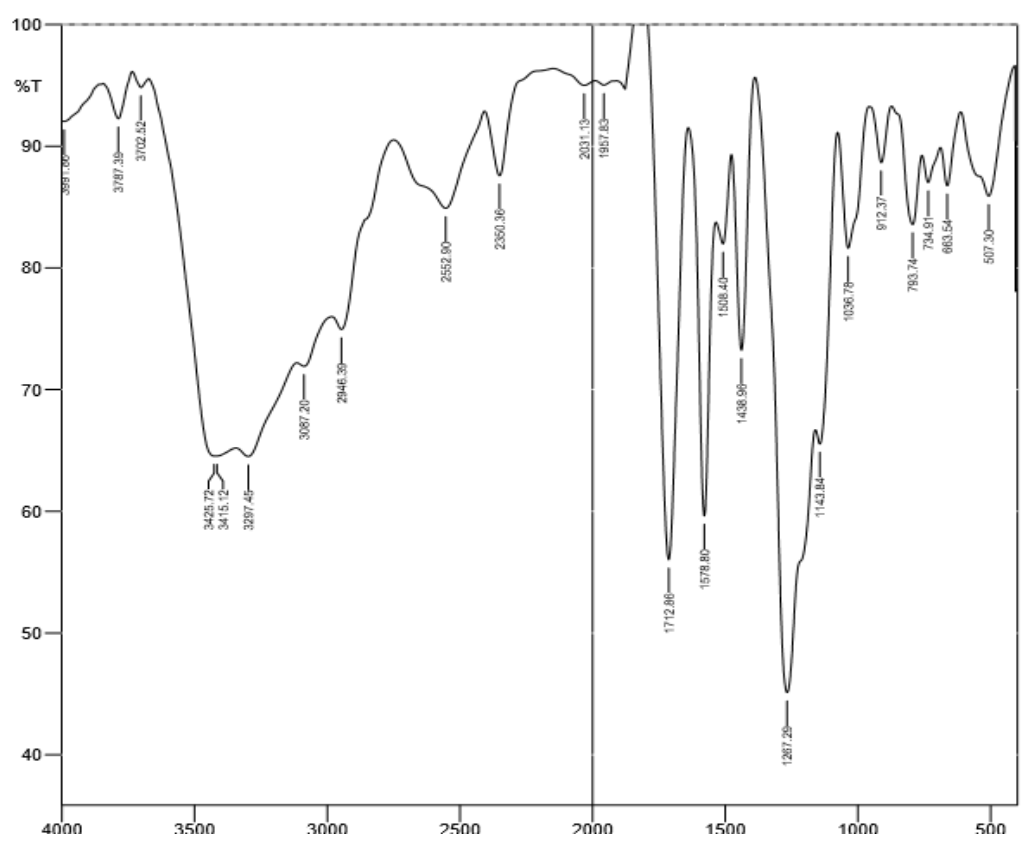

Figure 1. FT-IR spectrum of copolyester PHSI

The FT-IR spectra of all the six copolyesters unveils a characteristic stretching frequency in the range of $1712 \mathrm{~cm}^{-1}$ and $1754 \mathrm{~cm}^{-1}$ which may be due to ester carbonyl stretching frequency which clearly depicts the formation of the copolyester ${ }^{21}$.

\section{${ }^{1}$ H NMR Spectra}

The NMR spectra were recorded with BRUKER AV III $500 \mathrm{MHz}$ NMR instrument in DMSO-d6 solvent to make out the structural units present in the copolyester main chain. The ${ }^{1} \mathrm{H}$ NMR spectrum of copolyester PHOI is presented in Figure 2. The multiplet resonance signals at 7.10 to $7.67 \mathrm{ppm}$ are due to aromatic and olefinic protons. The methoxy protons in the chalcone moiety are represented in the range of 3.49 to $3.73 \mathrm{ppm}$. Methoxy protons of isovanillin moiety are observed in the range of 3 to $3.4 \mathrm{ppm}$. The polymers show splitting of multiplet in the range $7 \mathrm{ppm}$ to $8 \mathrm{ppm}$. This is due to the presence of more number of aromatic protons in different chemical environments.

\section{${ }^{13}$ C NMR Spectra}

${ }^{13} \mathrm{C}$ NMR spectrum of copolyester PHSI is represented in Figure 3. The resonance signals at 189.48 and $170.68 \mathrm{ppm}$ are assigned to ketone and ester carbons respectively. The aromatic carbon attached to ester oxygen atom is observed at $156.44 \mathrm{ppm}$. Chemical shifts of other aromatic and ethylenic group carbons are observed between 145.99 and $121.55 \mathrm{ppm}^{22}$.

\section{Structure and second harmonic generation property relationship}

The shape of the chalcone derivatives can be considered as lambda shape ( $\lambda$-shape); which helps to align the molecule in parallel head to tail alignment in the crystal packing ${ }^{23}$. Such molecular alignment usually exhibits high SHG efficiency. The presence of strong electron donating groups, the parallel head to tail alignment, inter and intra molecular hydrogen bonds are the reasons to get high SHG efficiency. The essential factor for efficient SHG is that the molecules are arranged in parallel or zig- zag head-to-tail fashion with molecules interlinked 
by strong hydrogen bond interactions ${ }^{24}$. These hydrogen bond interactions are the essential line to extend the molecular charge transfer into supra- molecular realm and there by extending the conjugation throughout the interaction length of the crystal. Such an increase in the conjugation via hydrogen bond interactions leads to the very high SHG efficiency of molecular crystals $^{25,26}$. The D (donor)-H... X (acceptor), $\pi \ldots \pi$ type interactions are believed to contribute significantly to the macroscopic NLO properties of organic crystals in the exited state $^{25}$. In the synthesized bis-chalcone polymers D- $\pi$-A- $\pi$-D type interactions are present and hence significant NLO property is observed in these polymers. Effective charge transfer depends on the molecular planarity ${ }^{27}$ and a twist in molecule may result in an ineffective charge transfer leading to decreased NLO response. Thus the high SHG efficiency of polymers PHSI, PHGI and PHOI might be attributed to the greater flow of electrons whereas the polymers PCSI, PCGI and PCOI exhibit low SHG efficiency when compared with respect to KDP due to their disturbed planar structure.

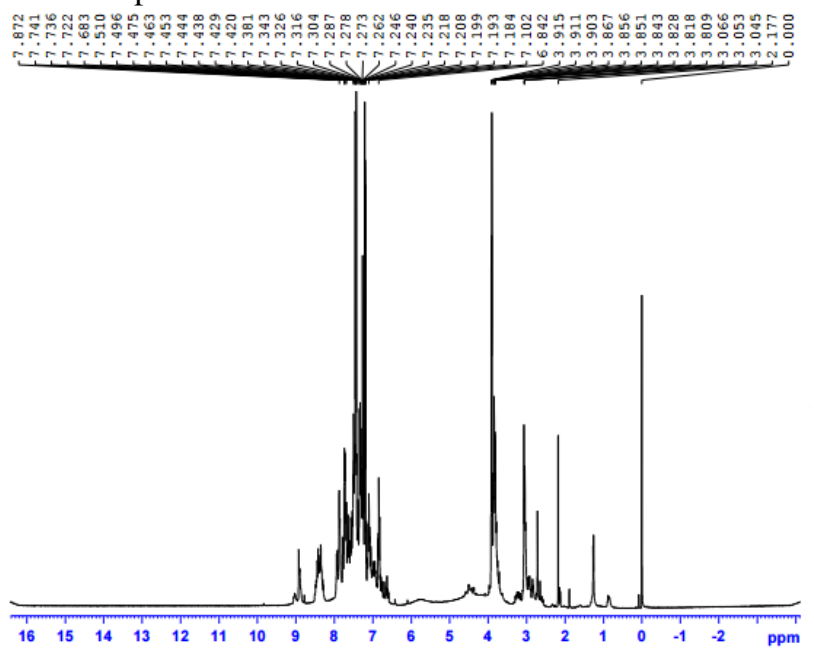

Figure 2. ${ }^{1} \mathrm{H}$ NMR spectrum of copolyester PHOI

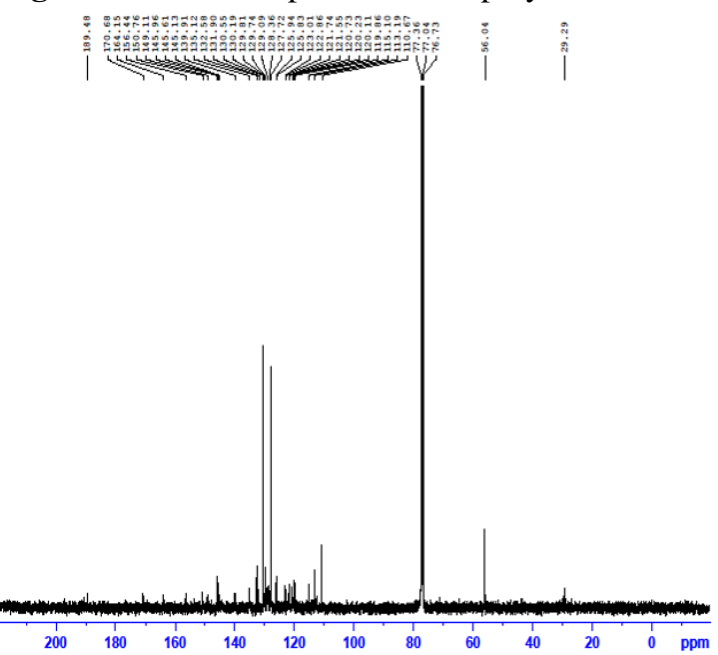

Figure 3. ${ }^{13} \mathrm{C}$ NMR Spectrum of copolyester PHSI 
Thermo gravimetric analysis (TGA)

The thermal transitions of the copolyesters were studied in the powder form by recording the TGA response curve in the temperature range $30{ }^{\circ} \mathrm{C}$ to $1000{ }^{\circ} \mathrm{C}$, at a rate of $10{ }^{\circ} \mathrm{C} / \mathrm{min}$, in an inert nitrogen atmosphere using TGA Q500 V20.10 Build 36 instrument. Figures 4, 5 and 6 represent the TGA thermograms of the copolyesters namely PHSI, PHGI and PHOI respectively.

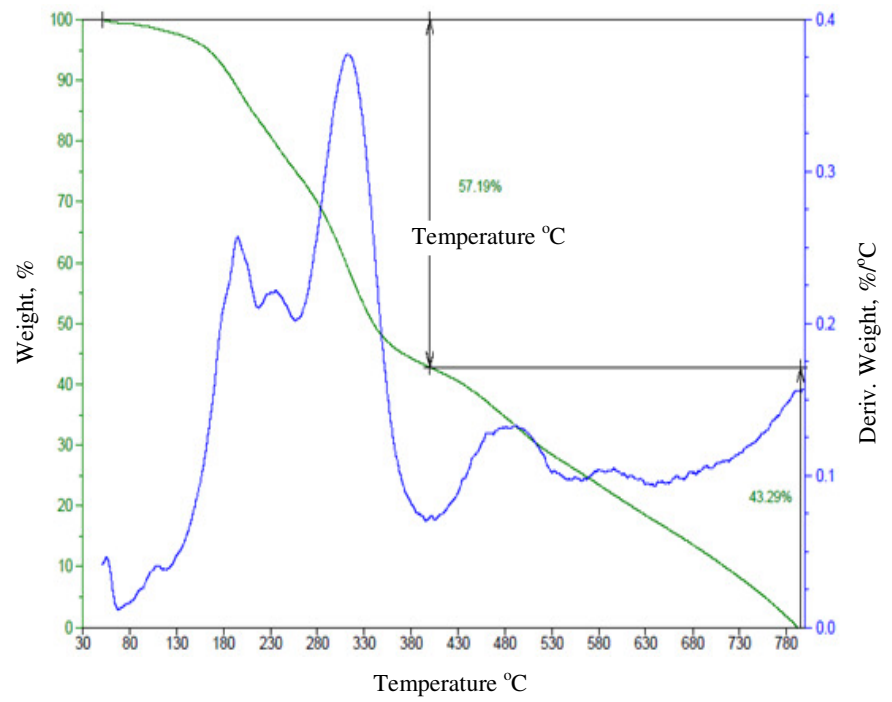

Figure 4. TGA thermogram of copolyester PHSI

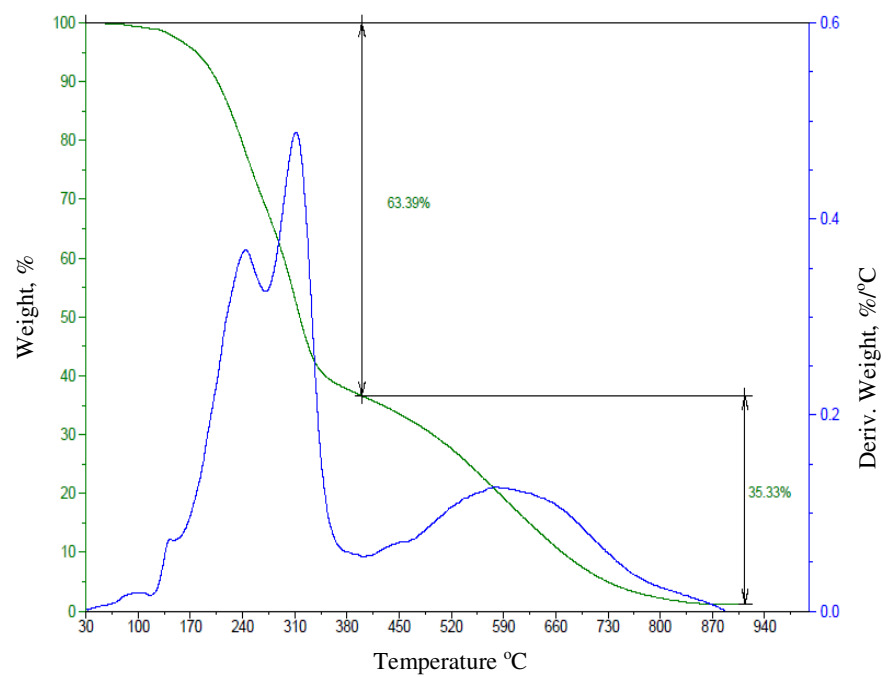

Figure 5. TGA thermogram of copolyester PHGI

The initial decomposition temperature (IDT) of the copolyesters C4, C5 and C6 are $124{ }^{\circ} \mathrm{C}$, $147{ }^{\circ} \mathrm{C}$ and $112{ }^{\circ} \mathrm{C}$ respectively. The copolyesters show $50 \%$ weight loss at temperatures $339{ }^{\circ} \mathrm{C}, 319^{\circ} \mathrm{C}$ and $337{ }^{\circ} \mathrm{C}$ proves flame retardancy and stability of the PHSI, PGHI and PHOI. The thermal study reveals that the copolyesters possess good thermal stability. 


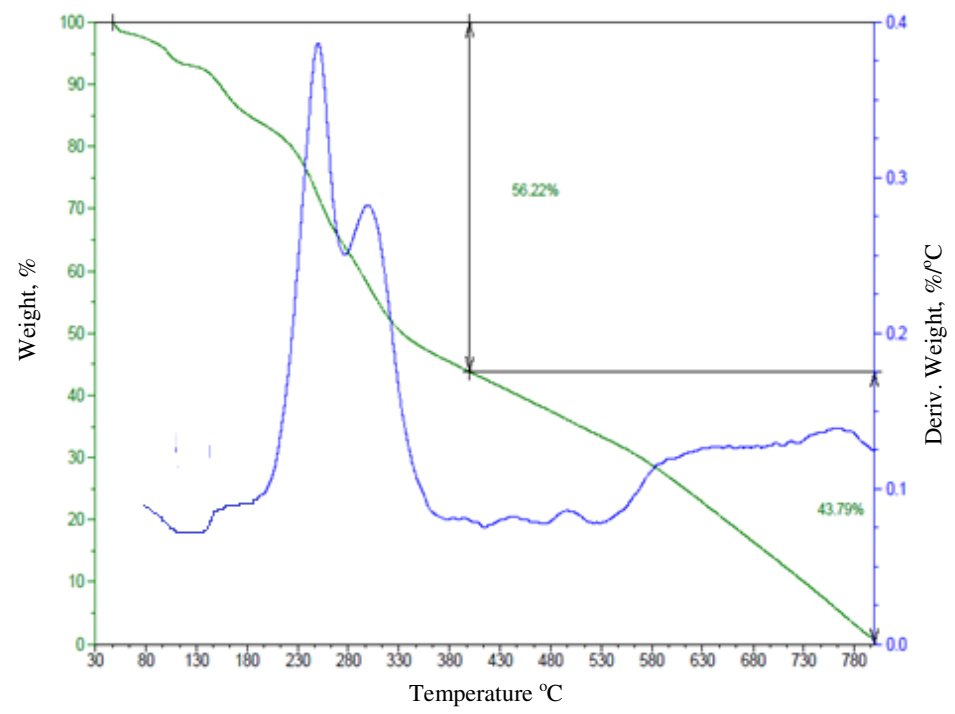

Figure 6. TGA thermogram of copolyester PHOI

\section{Measurement of SHG efficiency}

The SHG efficiencies of the chalcone based copolyesters were evaluated with the powder method developed by Kurtz and Perry ${ }^{28}$. The powder sample was packed in a microcapillary of uniform bore and exposed to laser radiations of a Q-switched Nd:YAG laser beam $(1064 \mathrm{~nm}, 1.2 \mathrm{~mJ}, 10 \mathrm{~ns}, 10 \mathrm{~Hz})$. The SHG was confirmed by the emission of green radiation. The amplitude of the SHG output voltage was measured using photomultiplier and digitalizing oscilloscope assembly. Sample of KDP and urea were used as reference material for the current measurement ${ }^{29}$. The SHG efficiencies of the copolyesters PCSI, PCGI and PCOI are $45 \mathrm{mV}, 60 \mathrm{mV}$ and $40 \mathrm{mV}$ respectively compared with $\mathrm{KDP}(55 \mathrm{mV})$. The SHG efficiencies of the copolyesters PHSI, PHGI and PHOI are $112 \mathrm{mV}, 97 \mathrm{mV}$ and $102 \mathrm{mV}$ respectively compared with $\mathrm{KDP}(102 \mathrm{mV})$.

\section{Conclusion}

Six second order nonlinear optical chalcone copolyesters were synthesized and characterized. Among the six copolyesters, PHSI is found to possess efficiency more than that of KDP under the same conditions. The copolyesters have better thermal stability. Spectral data reveals the molecular structural identity of the synthesized materials. The results exemplify that all the chalcone copolyesters are good NLO materials for second harmonic generation. Donor-acceptor substituted chalcones appears to be excellent scaffold suitable for exploring new NLO materials.

\section{Acknowledgement}

We acknowledge the Instrumentation Centre of Ethiraj College for Women for providing us the FT-IR spectral data.

\section{References}

1. Chang D E, Vuletic V and Lukin M D, Nat Photonics, 2014, 8, 685-694; DOI:10.1038/nphoton.2014.192 
2. Uchida K, Kaneko S, Omi S, Hata C, Tanzi H, Asahara Y, Ikushima A J, Tokizaki T and Nakumara, $J$ Opt Soc Am B, 1994, 11(7), 1236-1243; DOI:10.1364/JOSAB.11.001236

3. Bosshard C, Spreiter R and Gunter P J, Opt Soc Am B, 2001, 18(11), 1620-1626; DOI:10.1364/JOSAB.18.001620

4. Collini E, Mazzucato S, Zerbetto M, Ferrante C, Bozio R, Pizzotti M, Tessore F and Ugo F, Chem Phy Lett., 2008, 454(1-3), 70-74; DOI:10.1016/j.cplett.2008.01.054

5. John Kiran A, Chandrasekharan K, Satheesh Rai Nooji, Shashikala, H D and Umesh G, Chem Phys., 2006, 324, 699-704; DOI:10.1016/j.chemphys.2005.12.006

6. Arivazhagan M and Senthil Kumar J, Ind J Pure Appl Phys., 2012, 50(6), 363-373.

7. D'Silva E D, Krishna Podagattlapalli G, Venugopal Rao S and Dharma Prakash S M Mat Res Bull., 2012, 47(11), 3552-3557; DOI:10.1016/j.materresbull.2012.06.063

8. Janardhana K, Ravindrachary V, Rajesh Kumar P C, Umesh G, Manjunatha K B and Ismayil, Ind J Pure App Phys., 2013, 51, 844-850.

9. Shettigar S, Umesh G, Chandrasekharan K, Sarojini B K and Narayana B, Opt Mater., 2008, 30, 1297-1303; DOI:10.1016/j.optmat.2007.06.008

10. Ravindra H J, John Kiran A, Chandrasekharan K, Shashikala H D and Dharamprakash S M, App Phy B Lasers Opt., 2007, 88(1), 105-110; DOI:10.1007/s00340-007-2677-8

11. Hornak L H, Polymers for Light Wave and Integrated Optics; Marcel Dekker: New York, 1992

12. Marius Albota, David Beljonne, Jean Luc Brebas, Jaffrey Ehrlich E, Jia Ying Fu A, Ahmed Heikal E, Samuel Hess, Thierry Kogej, Michael Levin D, Seth Marder R, Mc Cord Maughon D, Joseph Perry W, Harald Rockel, Rumi M, Subramaniam G, Watt Webb $W$ and Wu L X, Science, 1998, 281(5383), 1653-1656; DOI:10.1126/science.281.5383.1653

13. Reinhardt B A, Brott L L, Clarson S J, Dillard A G, Bhatt J C, Kannan R, Yuan L, He Q S and Prasad P N, Chem Mater., 1998, 10(7), 1863-1874; DOI:10.1021/cm980036e

14. Zhao B, Lu W Q, Zhou Z H and Wu Y, J Mat Chem., 2000, 10, 1513-1517; DOI:10.1039/A909757K

15. Poornesh P, Seetharam Shettigar, Umesh G, Manjunatha K B, Kamath K P, Sarojini B K and Narayana B, Opt Mat., 2009, 31(5), 854-859; DOI:10.1016/j.optmat.2008.09.007

16. Poornesh P, Umesh G, Ravi K, Manjunatha K B, Hegde P K, Manjunatha M G and Adhikari A V, ICOP Int. Con. On Optics and Photonics, Chandigarh, India, 2009.

17. Chitra M, Reuben Jonathan D, Rajan Y C and Duraipandiyan V, Int J Chem Appl., 2013, 5(2), 73.

18. Arumugasamy E, Baskar B and Kannappan V, J Polym Mater., 2000, 17, 4.

19. Sidharthan J, Reuben Jonathan D and Peter Amaladhas T, Int J Chem Appl., 2012, 4(3), 241-250.

20. Perundevi T S, Reuben Jonathan D and Kothai S, Int J Adv Res., 2015, 3, 1147-1154.

21. Lakshmi Devi D, Reuben Jonathan D and Kothai S, J Chem Pharm Res., 2016, 8(3), 1041-1046.

22. Balaji R and Nanjundan S, React Func Polym., 2001, 49(1), 77-86;

DOI:10.1016/S1381-5148(01)00062-1

23. Sutherland R L, McLean D G and, Kirkpatrick S, Handbook of Nonlinear Optics; $2^{\text {nd }}$ Edition, Marcel Dekker: New York.

24. Munn R W and Ioronside C N, Principles and Appli- cations of Nonlinear Optical Materials; Chapman and Hell: London, 1993. 
25. Cole J M, Howard J A K and Intyre G J, Acta Crystallographica B, 2001, 57, 410414; DOI:10.1107/S0108768101002154

26. Tam W, Guerin B, Calabrese J C and Stevenson S H, Chem Phy Lett., 1989, 154(2), 93-96; DOI:10.1016/S0009-2614(89)87265-3

27. Zyss J, (Eds), Molecular Nonlinear Optics; Materials, Physics and Devices. Academic Press: New York, 1994.

28. Kurtz S K and Perry T T, J App Phy., 1968, 39, 3798-3813; DOI:10.1063/1.1656857

29. Patil P S, Dharmaprakash S M, Ramakrishna K, Hoong K F, Sai Santosh Kumar R and Narayana Rao D, J Cry Grow., 2007, 303(2), 520-524; DOI:10.1016/j.jcrysgro.2006.12.068 